\title{
Correction to: Crossed-Time Delay Neural Network for Speaker Recognition
}

\author{
Liang Chen, Yanchun Liang, Xiaohu Shi, You Zhou, \\ and Chunguo $\mathrm{Wu}$
}

\section{Correction to: \\ Chapter "Crossed-Time Delay Neural Network for Speaker Recognition" in: J. Lokoč et al. (Eds.): MultiMedia Modeling, LNCS 12572, https://doi.org/10.1007/978-3-030-67832-6_1}

The book was inadvertently published with a typo in the third author's name "Xiaoshu Shi", i.e., an extra "s" was added whereas it should have read "Xiaohu Shi". This has been now corrected with the erratum. 